\title{
Products from NASA’s In-Space Propulsion Technology Program Applicable to Low-Cost Planetary Missions
}

\author{
David J. Anderson ${ }^{1}$, David.J.Anderson@nasa.gov \\ Eric Pencil1, Eric.J.Pencil@nasa.gov \\ Daniel Vento', Daniel.M.Vento@nasa.gov \\ Todd Peterson ${ }^{1}$, Todd.T.Peterson@nasa.gov \\ John Dankanich², John.Dankanich@nasa.gov \\ David Hahne ${ }^{3}$, David.E.Hahne@nasa.gov \\ Michelle M. Munk ${ }^{3}$, Michelle.M.Munk@nasa.gov \\ ${ }^{1}$ NASA Glenn Research Center, 21000 Brookpark Road, Cleveland, OH 44135 \\ ${ }^{2}$ Gray Research Inc., 21000 Brookpark Road, Cleveland, OH 44135 \\ ${ }^{3}$ NASA Langley Research Center, 1 North Dryden Street, Hampton, VA 23681
}

\begin{abstract}
Since September 2001 NASA's In-Space Propulsion Technology (ISPT) program has been developing technologies for lowering the cost of planetary science missions. Recently completed is the high-temperature Advanced Material Bipropellant Rocket (AMBR) engine providing higher performance for lower cost. Two other cost saving technologies nearing completion are the NEXT ion thruster and the Aerocapture technology project. Also under development are several technologies for low cost sample return missions. These include a low cost Hall effect thruster (HIVHAC) which will be completed in 2011, light weight propellant tanks, and a Multi-Mission Earth Entry Vehicle (MMEEV). This paper will discuss the status of the technology development, the cost savings or performance benefits, and applicability of these in-space propulsion technologies to NASA's future Discovery, and New Frontiers missions, as well as their relevance for sample return missions.
\end{abstract}

Keywords: electric and chemical propulsion, aerocapture, entry vehicles, trajectory tools

\section{Introduction}

NASA's Science Mission Directorate (SMD) missions seek to answer important science questions about our planet, the Solar System and beyond. To meet NASA's future mission needs, the goal of the ISPT program is the development of new enabling propulsion technologies that cannot be reasonably achieved within the cost or schedule constraints of mission development timelines. Since 2001, the InSpace Propulsion Technology (ISPT) program has been developing in-space propulsion technologies that will enable and/or benefit near and mid-term NASA robotic science missions by significantly reducing cost, mass, and/or travel times. ISPT technologies will help deliver spacecraft to SMD's destinations of interest.

An objective of ISPT is to develop products that realize near-term and mid-term benefits. The program primarily focuses on technologies in the mid TRL range (TRL 3 to $6+$ range) that have a reasonable chance of reaching maturity in 4-6 years. The objective is to achieve technology readiness level (TRL) 6 and reduce risk sufficiently for mission infusion. The project strongly emphasizes developing propulsion products for NASA flight missions, that will be ultimately manufactured by industry and made equally available to all potential users for missions and proposals.

The ISPT program is currently developing technology in four areas. These include Advanced Chemical and Electric Propulsion, Entry Vehicle Technologies, Sample Return Propulsion, and Systems/Mission Analysis. These in-space propulsion technologies are applicable, and potentially enabling for future NASA Discovery, New Frontiers, and sample return missions currently under consideration, as well as having broad applicability to potential Flagship missions. For more background on ISPT, please see references [1] and [2]. 


\section{Technology Relevance}

The ISPT priorities and products are tied closely to the science roadmaps, the SMD's science plan, and the decadal surveys. ISPT therefore emphasizes technology development with mission pull. In 2006, the Solar System Exploration (SSE) Roadmap [3] identified technology development needs for Solar System exploration, and described transportation technologies as highest priority, with the highest priority propulsion technologies being electric propulsion and aerocapture. Excerpts from the science community are discussed in Ref. [4]. Initially, ISPT's responsibility was to develop technologies for Flagship missions, but in 2006 the focus evolved to technology investments that would also be applicable to New Frontiers and Discovery competed missions. So, aerocapture (the use of aerodynamic drag for orbit capture) and electric propulsion continued to be a priority, but the refocus activity also recommended a long-life lower power Hall system.

Looking towards ISPT's future, the 2011 Planetary Science Decadal Survey [13] was released March 2011 and will provide guidance for ISPT's future technology investments. The Decadal Survey made many references to ISPT technologies like aerocapture, NEXT, AMBR, and astrodynamics, mission trajectory and planning tools. So this Decadal Survey is validating the technology investments ISPT has been making over the last 10 years, but also provides ISPT with a new focus for the next 10 to 20 years.

The Decadal Survey supported NASA developing a multi-mission technology investment program that will "preserve its focus on fundamental system capabilities rather than solely on individual technology tasks." The Decadal Survey highlighted the NEXT system development as such an example of this "integrated approach" of "advancement of solar electric propulsion systems to enable wide variety of new missions throughout the solar system." The Decadal Survey also recommends "making similar equivalent systems investments" in the advanced Ultraflex solar array technology and aerocapture. The Decadal Survey also discussed the importance of developing those system technologies to TRL6.

One recommendation in the Decadal Survey was for "a balanced mix of Discovery, New Frontiers, and Flagship missions, enabling both a steady stream of new discoveries and the capability to address larger challenges like sample return missions and outer planet exploration." These broad mission needs would in turn require a balanced set of multi-mission technologies and integrated system capabilities. The Survey acknowledges that a "robust Discovery and New Frontiers program would be substantially enhanced by such a commitment to multi-mission technologies."

\section{Aerocapture}

Aerocapture is the process of entering the atmosphere of a target body to practically eliminate the chemical propulsion requirements of orbit capture. Aerocapture is the next step beyond aerobraking, which relies on multiple passes high in the atmosphere using the spacecraft's drag to reduce orbital energy. Aerobraking has been used at Mars on multiple orbiter missions. Aerocapture, illustrated in Figure 1, maximizes the benefit from the atmosphere by capturing into orbit in a single pass. Aerocapture represents a major advance over aerobraking techniques, by flying at a lower altitude where the atmosphere is more dense. Keys to successful aerocapture are accurate arrival state knowledge, validated atmospheric models, sufficient vehicle control authority (i.e. lift-to-drag ratio), and robust guidance during the maneuver. A lightweight thermal protection system and structure will maximize the aerocapture mass benefits.

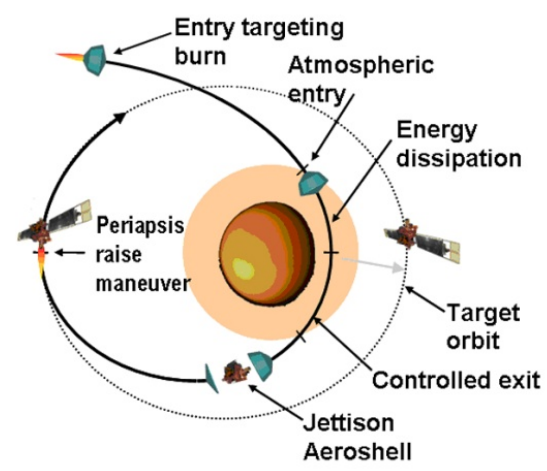

Figure 1. Illustration of the aerocapture maneuver.

Executing the aerocapture maneuver itself is what enables the great mass savings over other orbital insertion methods. If the hardware subsystems are not mass efficient, or if performance is so poor that additional propellant is needed to adjust the final orbit, the benefits can be significantly reduced. ISPT efforts in aerocapture subsystem technologies are focused on improving the efficiency and number of suitable alternatives for aeroshell structures and ablative thermal protection systems (TPS). These include development of families of low and medium density (14-36 lbs/ $\left.\mathrm{ft}^{3}\right)$ TPS and the related sensors, 
development of a carbon-carbon rib-stiffened rigid aeroshell, and high temperature honeycomb structures and adhesives. Development occurred on inflatable decelerators through concept definition and initial design and testing of several inflatable decelerator candidates. Finally, progress has been made through improvement of models for atmospheres, aerothermal effects, and algorithms and testing of a flight-like guidance, navigation and control (GN\&C) system.

Aerocapture has been proven repeatedly in detailed analyses to be an enabling or strongly enhancing technology for several atmospheric targets. The ISPT project team continues to mature aerocapture component in preparation for a flight demonstration, and rapid aerocapture analysis tools are being developed and made available to a wider user community. The TPS materials developed through ISPT enhance a wide range of missions by reducing the mass of entry vehicles. Some of the remaining gaps for technology infusion are efficient TPS for Venus and high-speed Earth return. All of the other component subsystems for an aerocapture vehicle are currently at or funded to reach TRL 6 in the next year. This assessment of technology readiness is detailed in Ref. [5]. The structures and TPS subsystems as well as the aerodynamic and aerothermodynamic tools and methods can be applied to small-scale entry missions even if the aerocapture maneuver is not utilized.

The Aerocapture system cannot reach TRL 6 without space flight validation, since it is impossible to match the flight environment in ground facilities. This validation can be accomplished by utilizing Aerocapture on a science mission, or by a dedicated space flight validation experiment. NASA's Science Mission Directorate has incentivized the use of Aerocapture in its recent Discovery Announcement of Opportunity. Since a Discovery mission utilizing Aerocapture was not selected, Aerocapture will likely seek other opportunities to be validated in space. A space flight validation is expensive, but the costs will be recouped very quickly if just one mission's launch vehicle cost is reduced as a result of the lower mass requirement enabled by Aerocapture. The validation immediately reduces the risk to the first user and matures the maneuver for application to multiple, potentially lower-cost, missions to Titan, Mars, Venus, and Earth. Moreover, once Aerocapture is proven a reliable tool, it is anticipated that entirely new missions will become possible. Additional information on Aerocapture technology developments can be found in the Discovery program library [6]. Using Aerocapture, significant cost benefits are realized for multiple missions. When the overall system mass is reduced, the mission can utilize a smaller launch vehicle, saving tens of millions of dollars. Detailed mission assessment results can be found in the Aerocapture-related references in Ref. [2].

\section{Multi-Mission Earth Entry Vehicle (MMEEV)}

The Multi-Mission Earth Entry Vehicle (MMEEV) is a flexible design concept which can be optimized or tailored by any sample return mission, including lunar, asteroid, comet, and planetary (e.g. Mars), to meet that mission's specific requirements. Based on the Mars Sample Return (MSR) Earth Entry Vehicle (EEV) design, which due to planetary protection requirements, is designed to be the most reliable space vehicle ever flown, the MMEEV concept provides a logical foundation by which any sample return mission can build upon in optimizing an EEV design which meets their specific needs. By leveraging common design elements, this approach can significantly reduce the risk and associated cost in development across all sample return missions, while also providing significant feed-forward risk reduction in the form of technology development, testing, and even flight experience, for an eventual MSR implementation.

The current MMEEV parametric configuration is presented in Figure 2 (basic vehicle architecture). Since each individual sample return mission may have a unique set of performance metrics of highest interest, the goal is to provide a qualitative performance comparison across a specified trade space. From this, each sample return mission can select the most desirable design point from which to begin a more optimized design.

Continued development of the MMEEV models is planned to include: more sophisticated parametric configuration, including payload accommodation, models; higher fidelity impact dynamics model (e.g. finite-element model); updated aerodynamics models based on ground (e.g. wind tunnel and ballistic range) testing as well as Computational Fluid Dynamics (CFD) analysis; and high fidelity TPS mass/thickness sizing models for additional candidate TPS materials. MMEEV performance studies will also continue, with the eventual integration of the MMEEV models into a prototype EDL analysis tool, originally developed in support of ISPT aerocapture studies, and currently being developed to support mission studies to any celestial body with an atmosphere. 


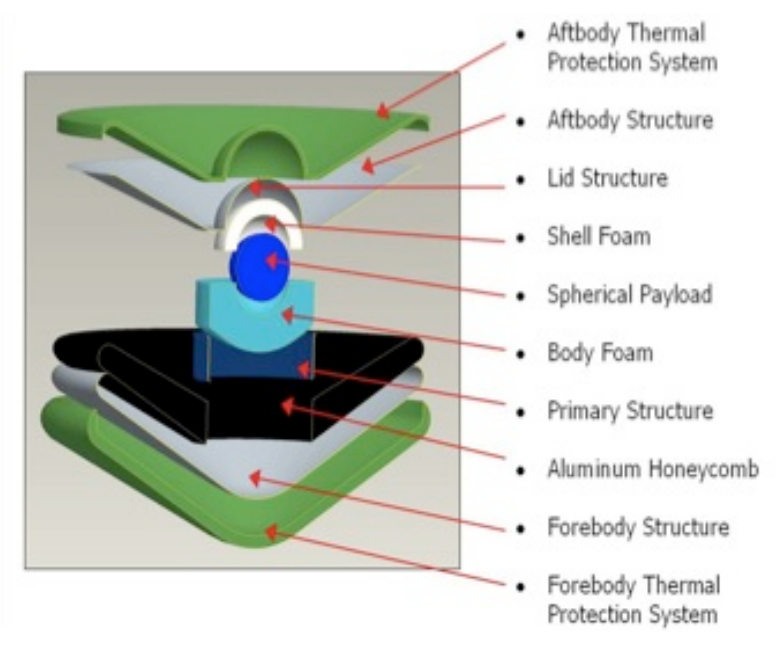

Figure 2. Basic MMEEV architecture

The biggest challenge for any space vehicle, including the MMEEV, is to adequately prove the reliability of the components, subsystems, and the flight system as a whole. The current estimate to develop the EEV technology for MSR to TRL-6 is approximately $\$ 41$ million. This does not include a dedicated flight test, which many experts agree is needed to achieve the $10^{-6}$ probability of failure, since the entry flight environment cannot be replicated in ground-based facilities. One way to achieve a flight validation for little extra cost to NASA is to use the MMEEV design concept, or at least the major components of the design, in sample return missions likely to fly prior to MSR, such as New Frontiers or Discovery. NASA Headquarters managers and the InSpace Propulsion Technology (ISPT) team are pursuing this approach, but currently there are no manifested missions that are planning to use an MSR EEV design.

\section{Solar Electric Propulsion (S EP)}

Solar Electric Propulsion (SEP) enables missions requiring large post launch $\Delta \mathrm{V}$. SEP has applications to rendezvous and sample-return missions to small bodies and fast trajectories towards the outer planets.

Electric propulsion is both an enabling and enhancing technology for reaching a wide range of targets. The high specific impulse, or efficiency of electric propulsion system, allows direct trajectories to multiple targets that are chemically infeasible. The technology allows for rendezvous missions in place of fly-bys, and as planned in the Dawn mission can enable multiple destinations.

This technology offers major performance gains, only moderate development risk, and has significant impact on the capabilities of new missions. Current plans include completion of the NASA's
Evolutionary Xenon Thruster (NEXT) Ion Propulsion System target at Flagship, New Frontiers and demanding Discovery missions.

The GRC-led NEXT project was competitively selected to develop a nominal $40-\mathrm{cm}$ gridded-ion electric propulsion system. [2] The objectives of this development were to improve upon the state-of-art (SOA) NASA Solar Electric Propulsion Technology Application Readiness (NSTAR) system flown on Deep Space-1 to enable flagship class missions by achieving the performance characteristics listed in Table 1.

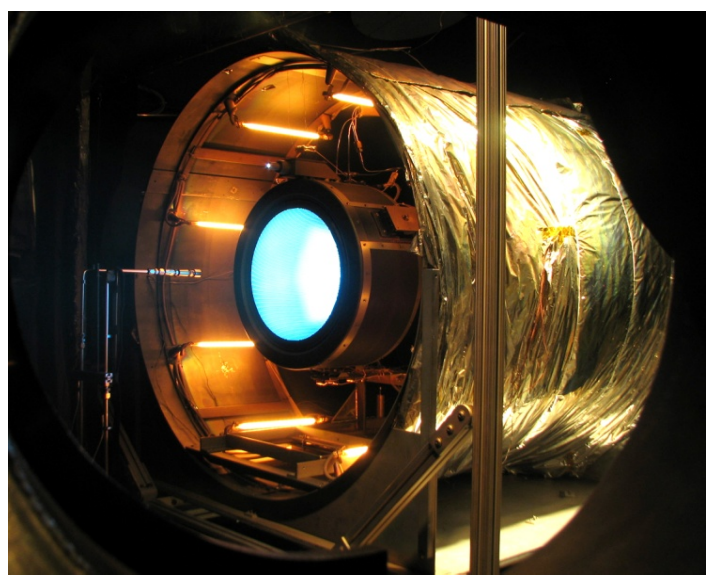

Figure 3. NEXT thermal vacuum testing at JPL

The ion propulsion system components developed under the NEXT task include the ion thruster, the power-processing unit (PPU), the feed system, and a gimbal mechanism. The NEXT project is developing prototype-model (PM) fidelity thrusters through Aerojet Corporation. In addition to the technical goals, the project has the goal of transitioning thruster-manufacturing capability with predictable yields to an industrial source. To prove out the performance and life of the NEXT thruster, a series of tests have, or are being, performed. The NEXT PM thruster completed a short duration test in which overall ion-engine performance was steady with no indication of performance degradation. A NEXT PM thruster has also passed qualification level environmental testing (Figure 3). As of April 30, 2011 the Long Duration Test (LDT) of the NEXT engineering model (EM) thruster achieved over 611$\mathrm{kg}$ xenon throughput, $23.0 \times 10^{6} \mathrm{~N}$-s of total impulse, and $>36,000$ hours at multiple throttle conditions. The NEXT LDT wear test demonstrates the largest total impulse ever achieved by a gridded-ion thruster. ISPT funding for the thruster life test continues through FY12 with the aim of demonstrating up to $750 \mathrm{~kg}$ of xenon throughput. [7] 
The NEXT thruster has clear mission advantages for very challenging missions. For example, the Dawn Discovery Mission only operates one NSTAR thruster at a time, but requires a second thruster for throughput capability. For the same mission, the NEXT thruster could deliver mass, equivalent to doubling the science package, with only a single thruster. Reducing the number of thrusters reduces propulsion system complexity and spacecraft integration challenges. NEXT can enable a lower cost implementation through eliminating system complexity. Comparisons between the State-of-theArt (SOA) NSTAR thruster and the NEXT thruster are shown below in Table 2 .

Table 1. Performance comparison of NSTAR and NEXT ion thrusters

\begin{tabular}{|c|c|c|}
\hline Characteristic & $\begin{array}{l}\text { NSTAR } \\
\text { (SOA) }\end{array}$ & NEXT \\
\hline Max. Thruster Power (kW) & 2.3 & 6.9 \\
\hline Max. Thrust (mN) & 91 & 236 \\
\hline $\begin{array}{lll}\text { Throttle } & \text { Range } & \text { (Max./Min. } \\
\text { Thrust) }\end{array}$ & 4.9 & 13.8 \\
\hline Max. Specific Impulse (sec) & 3120 & 4190 \\
\hline Total Impulse $\left(\times^{10}{ }^{6} \mathrm{~N}-\mathrm{sec}\right)$ & $>5$ & $>18$ \\
\hline Propellant Throughput (kg) & 200 & 750 \\
\hline
\end{tabular}

The missions that are improved through the use of the NEXT thruster are those requiring significant post-launch $\Delta \mathrm{V}$, such as sample returns, highly inclined, or deep-space body rendezvous missions. The comet sample-return mission was studied for several destinations because of its high priority within the New Frontiers mission category. Electric propulsion enables a much wider range of feasible targets. Specifically for Temple 1 in Ref. [2] the NSTAR thruster is able to complete the mission, but requires large solar arrays and four or five thrusters to deliver the required payload. NEXT would be able to deliver 10 percent more total mass and require half the number of thrusters.

Additional information on the NEXT system can be found in the NEXT Ion Propulsion System Information Summary in the New Frontiers and Discovery program libraries. [6], [7], [8]

\section{Electric Propulsion for S ample Return and Discovery-class Missions}

ISPT is investing in Sample Return Propulsion technologies for applications such as Earth-Return
Vehicles for large and small bodies. The first example leverages the development of a HighVoltage Hall Accelerator (HIVHAC) Hall thruster into a lower-cost electric propulsion system. [1] HIVHAC is the first NASA electric propulsion thruster specifically designed as a low-cost electric propulsion option. It targets Discovery and New Frontiers missions and smaller mission classes. The HIVHAC thruster does not provide as high a maximum specific impulse as NEXT, but the higher thrust-to-power and lower power requirements are suited for the demands of some Discovery-class missions and sample return applications. Advancements in the HIVHAC thruster include a large throttle range from $0.3-3.5 \mathrm{~kW}$ allowing for a low power operation. It results in the potential for smaller solar arrays at cost savings, and a long-life capability to allow for greater total impulse with fewer thrusters. It allows for cost benefits with a reduced part count resulting in less complex and lower cost propulsion system.

Wear tests of the NASA-103M.XL thruster validated and demonstrated a means to mitigate discharge channel erosion as a life limiting mechanism in Hall thrusters. The thruster, shown in Figure 4, operated in excess of 5500 hours $(115 \mathrm{~kg}$ of xenon throughput) at a higher specific impulse (thruster operating voltage) as compared to SOA Hall thrusters.

Components for two Engineering Model (EM) thrusters were designed and fabricated. Preliminary performance mapping of the EM thruster at various operating conditions was performed at NASA Glenn Research Center (GRC). [1] In the future, the test sequence will include performance acceptance tests, environmental tests and a long duration test in FY11 and FY12. Current plans include the design, fabrication and assembly of a full Hall propulsion system, but are pending final approval to proceed.

In addition to the thruster development, the HIVHAC project is evaluating power processing unit (PPU) and xenon feed system XFS development options that were sponsored by other projects but can apply directly to a HIVHAC system. The goal is to advance the TRL level of a Hall propulsion system to level 6 in preparation for a first flight.

The functional requirements of a HIVHAC PPU are operation over a power throttling range of 300 to $3,800 \mathrm{~W}$, over a range of output voltages between 200 and $700 \mathrm{~V}$, and output currents between 1.4 and $5 \mathrm{~A}$ as the input varies over a range of 80 to $160 \mathrm{~V}$. A performance map across these demanding conditions was generated for one candidate option [1] that is being developed through NASA SBIR Program. 
Beyond conventional feed system options, one option for feed systems that was demonstrated with the Hall thruster is the advanced xenon feed system, developed by VACCO.

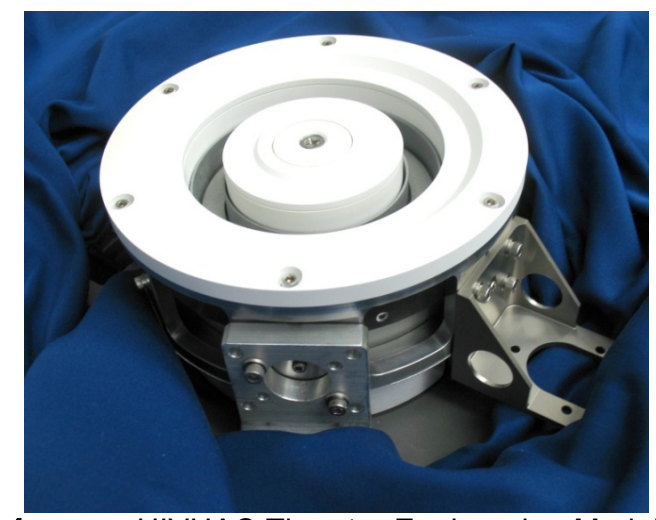

Figure 4

HIVHAC Thruster Engineering Model

For the Near-Earth Object (NEO) mission evaluated, the HIVHAC thruster system delivered over 30 percent more mass than the NSTAR system. The performance increase accompanied a cost savings of approximately 25 percent over the SOA NSTAR system. The Dawn mission was evaluated, and the expected HIVHAC Hall thruster delivered approximately 14 percent more mass at substantially lower cost than SOA, or decreasing the solar array provided equivalent performance at even greater mission cost savings. [1]

The second technology example of a Sample Return Propulsion Technology is the BPT-4000 hall thruster development. ISPT has invested in a life-test extension of the thruster to improve total impulse demonstrated capabilities. Also under evaluation is the operation of this thruster design at higher operating voltages, which improve thruster specific impulse. There are mission studies that indicate that BPT-4000 is directly applicable to ERV and Discovery-class missions.

\section{Propulsion Component Technologies}

ISPT also invests in the evolution of component technologies that offer significant performance improvements without increasing system level risk. Two component technologies currently receiving investments are xenon feed systems and Ultra-Light Tank Technology (ULTT).

ISPT is investing in the Advanced Xenon Feed System (AXFS) for electric propulsion systems. [1] The feed system is designed for an increased reliability combined with a decrease in system mass, volume, and cost as compared to SOA flight systems and comparable TRL 6 technology. The final development module, the pressure control module
(PCM), was completed in 2007. The Naval Research Laboratory (NRL) completed functional and environmental testing of the VACCO PCM in September of 2008. Following the environmental testing, the PCM was integrated with the FCMs and an integrated AXFS with controller was delivered to the project. NASA GRC completed hot-fire testing of the AXFS with the HIVHAC Hall thruster successfully demonstrating hot-fire operation using closed-loop control with downstream pressure feedback and with the Hall thruster discharge current. Follow-on testing will determine the viability of the AXFS to perform single-stage, single module, control from high-pressure xenon directly to a thruster.

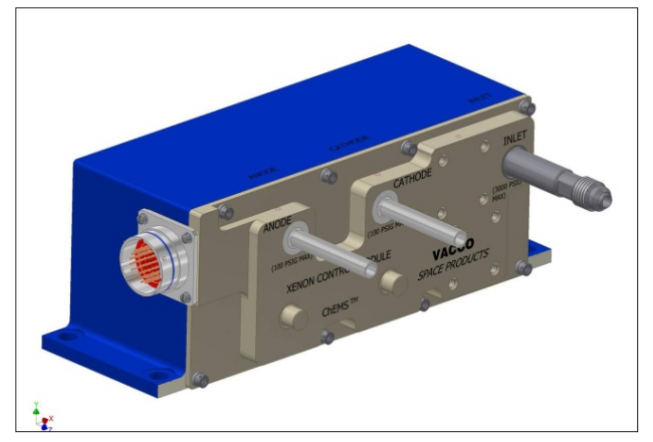

Figure 5. VACCO Xenon Flow Control Module

The AXFS technology is ready for transition into a qualification program. It achieves its objective [1] by demonstrating accurate xenon control with significant system reduction in mass and volume through the use of integrated modules for low-cost control options and/or reliability beyond practical SOA technology implementation. The resultant feed system represents a dramatic improvement over the NSTAR flight-feed system and represents an additional 70 percent reduction in mass, 50 percent reduction in footprint, and 50 percent reduction in cost over the baseline NEXT feed system at TRL 6 . The project successfully completed the integrated system testing and advanced the modules to TRL 6 . [2] A conceptual drawing of the Hall module is shown in Figure 5.

ISPT had previously invested in ultra lightweight tank technology (ULTT), which lead to flight tanks sized for but ultimately not used on the Mars Exploration Rover (MER) mission. The ULTT efforts in the past have focused on manufacturability and non-destructive evaluation of the lightweight tanks. Previous work on the lightweight propellant-tanks will continue with general applicability to all future planetary spacecraft. The mass savings, and resultant cost impacts, could be significant for the tank developments, since tanks are one of the largest 
spacecraft bus components. The ISPT project is currently planning to develop and qualify positive expulsive ultra light-weight tanks specifically for the MSL Sky Crane. These tanks can offer mass savings on the order of $24-30 \mathrm{~kg}$, dependent on the final tank wall thickness, and therefore increase the landed mass capability of Sky Crane for a relatively low cost per kg. While the tanks will be qualified for the Sky Crane application, the technology will be broadly applicable for a wide range of future low-cost science missions.

\section{Advanced Chemical Propulsion}

ISPT's approach to the development of chemical propulsion technologies is primarily the evolution of subcomponent technologies that still offers significant performance improvements, with minimal risk. The mission benefits in advanced chemical propulsion are synergistic, and the cumulative effects have tremendous potential. The infusion of the individual subsystems separately provides reduced risk, or combined provides considerable payload mass benefits. Ref [9] has a thorough description of the complete Advanced Chemical Propulsion effort that was concluded in 2009.

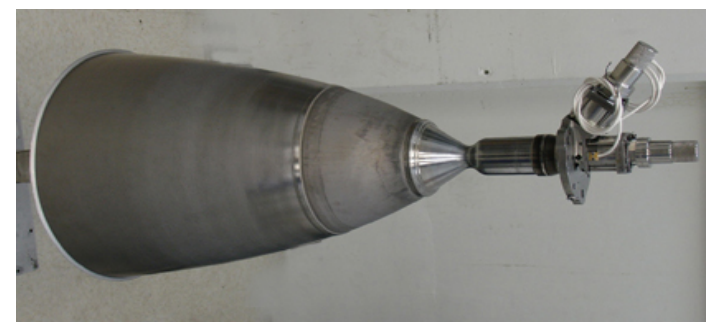

Figure 6. AMBR engine test article

The single largest investment within the advanced chemical propulsion technology area was the Advanced Materials Bipropellant Rocket (AMBR) engine (Figure 6), which was awarded, through a competitive process, to Aerojet Corporation in FY2006. The AMBR engine is a high temperature thruster that aimed to address cost and manufacturability challenges of using iridium coated rhenium chambers. The project [2] included the manufacture and hot-fire tests of a prototype engine demonstrating increase performance and validating new manufacturing techniques. Performance testing was conducted on the AMBR engine in October 2008 and February 2009 with long duration testing in June 2009. The thruster demonstrated an $I_{s p}$ of 333 seconds, which is the highest ever achieved for hydrazine/NTO (nitrogen tetroxide) propellant combination. The project also completed vibration, shock, and long duration testing to raise the TRL to 6. [10] Additional information is found in the AMBR information summary in the New Frontiers and Discovery program libraries. [6], [11]

\section{Systems/Mission Analysis}

Systems analysis is critical prior to investing in technology development. In today's environment, advanced technology must maintain its relevance through mission pull. The second focus of the systems analysis project area is the development and maintenance of tools for the mission and systems analyses. Improved and updated tools are critical to clearly understand and quantify mission and system level impacts of advanced propulsion technologies. Having a common set of tools increases confidence in the benefit of ISPT products both for mission planners as well as for potential proposal reviewers. Tool development efforts were completed on the Low-Thrust Trajectory Tool (LTTT) and the Advanced Chemical Propulsion System (ACPS) tool.

Low-thrust trajectory analyses are critical to the infusion of new electric propulsion technology. Lowthrust trajectory analysis is typically more complex than chemical propulsion solutions during the preliminary mission design phase. Some of the heritage tools prove to be extremely valuable, but cannot perform direct optimization and require good initial guesses by the users. This leads to solutions difficult to verify quickly and independently. The ability to calculate the performance benefit of complex electric propulsion missions is intrinsic to the determination of propulsion system requirements. The ISPT office invested in multiple low-thrust trajectory tools that independently verify low thrust trajectories at various degrees of fidelity.

ISPT products can ease technology infusion because of the ability for the user community to rapidly and accurately access the mission level impacts. In addition to the tools currently available, the ISPT project also sponsored the development of an Aerocapture quicklook tool to allow users an opportunity to quantify mission benefits of an aerocapture system including mass properties and geometry. Every effort will be made to have these tools validated, verified, and made publicly available. Instructions to obtain the tools currently available are provided on the ISPT project website. [12 ]

\section{Technology Infusion}

NASA recognizes that it is desirable to fly new technologies that enable new scientific investigations 
or to enhance an investigation's science return. The SSE Roadmap states that NASA will strive to maximize the payoff from its technology investments, either by enabling individual missions or by enhancing classes of missions with creative solutions. Discovery, New Frontiers, and Flagship missions potentially provide opportunities to infuse advanced technologies developed by NASA, and advance NASA's technology base and enable a broader set of future missions.

To benefit from its technology investments, NASA provided incentives for infusion of new technological capabilities that it had developed in the most recent New Frontiers and Discovery competed mission solicitations. The incentives for NEXT, AMBR, Aerocapture, and the Advanced Stirling Radioisotope Power System (ASRG) were in the form of increases to the cost cap for the mission, or providing the ASRG as Government Furnished Equipment (GFE). The Decadal Survey states "these technologies continue to be of high value to a wide variety of solar system missions." And that "NASA should continue to provide incentives for these technologies until they are demonstrated in flight." The 2011 Planetary Decadal Survey strongly supported continuing to incentivize these technologies until they are flown. [Ref 13] As funding and priorities allow, ISPT will also strive to maintain the capabilities associated with NEXT, AMBR, and aerocapture.

Beyond the New Frontiers and Discovery opportunities, ISPT continues to seek opportunities to infuse NEXT, AMBR, Aerocapture, and its other technologies into a wide range of possible future mission opportunities. The ISPT project office and NEXT team personnel are actively supporting various flagship science definition team (SDT) studies. See the ISPT Overview paper in the 2010 IEEE Aerospace Conference for more details regarding these studies. [2] [8] ISPT will continue to help in identifying the technology development that is required to accomplish the future missions being contemplated.

\section{Conclusion and Future Plans}

The ISPT program is investing in technologies to enhance or enable low-cost planetary science mission opportunities. The AMBR engine and Aerocapture investments are available for mission infusion. The NEXT ion propulsion system is planned to reach completion with the next year. The ISPT program is also progressing on a low-cost electric propulsion alternative. The program will continue to incentivize these technologies; reducing cost and risk of infusion to future low-cost mission opportunities.

\section{Acknowledgments}

The results and findings presented here are based on work funded by NASA's Science Mission Directorate (SMD). The ISPT program is managed out of the Glenn Research Center for SMD's Planetary Sciences Division (PSD).

\section{References}

[1] Anderson, D. J. et. al., "Sample Return Propulsion Technology Development under NASA's ISPT Project," 2011 IEEE Aerospace conference, March 2011, Paper \#1115

[2] Anderson, D. J. et. al., "In-Space Propulsion Technology Products for NASA's Future Science and Exploration Missions," 2011 IEEE Aerospace conference, March 20011, Paper \#1114.

[3] "2006 Solar System Exploration Roadmap for NASA's Science Mission Directorate," September 2006.

[4] Anderson, D. J. et. al., "The NASA In-Space Propulsion Technology Project, Products, and Mission Applicability," 2009 IEEE Aerospace conference, March 2009, Paper \#1176.

[5] Anderson, D. J. et. al., "The NASA In-Space Propulsion Technology Project's Current Products, and Future Directions," 2010 IEEE Aerospace conference, March 2010, Paper \#1078

[6] Discovery Program Library Web site http://discovery.larc.nasa.gov/dpl.html

[7] "NASA's Evolutionary Xenon Thruster (NEXT) Ion Propulsion system Information Summary Aug. 2008," New Frontiers Program Library Web site http://newfrontiers.larc.nasa.gov/NFPL.html

[8] New Frontiers Program Library Web site http://newfrontiers.larc.nasa.gov/NFPL.html

[9] Liou, L., et. al., "NASA In-Space Advanced Chemical Propulsion Development in Recent Years," 2010 IEEE Aerospace Conference, Big Sky, MT, March 6-13 2010.

[10]Henderson, S., et. al., "Performance Results for the Advanced Materials Bipropellant Rocket (AMBR) Engine," AIAA 2010-6883, 46 ${ }^{\text {th }}$ JPC, Nashville, TN, July 25-28, 2010.

[11] Advanced Material Bi-propellant Rocket (AMBR) Information Summary August 2008," New Frontiers Program Library Web site http://newfrontiers.larc.nasa.gov/NFPL.html

[12]NASA ISPT Web site http://spaceflightsystems.grc.nasa.gov/Advanced/ ScienceProject/ISPT/LTTT/.

[13] "Vision and Voyages for Planetary Science in the Decade 2013-2022," The National Academies Press, http://www.nap/edu, 2011 